\title{
Research on the equivalent elastic modulus of mechanical joint interface
}

\author{
Linlin Zhang ${ }^{1}$, Xiaoyang Li $^{1}$ and Yanjun Zeng ${ }^{2, a}$ \\ 1 College of Mechanical Engineering and Applied Electronics Technology, Beijing University of Technology, Beijing, \\ 100124, P.R. China \\ 2 Biomechanics and Medical Information Institute, Beijing University of Technology, Beijing 100022, P.R. China
}

Received 20 February 2016, Accepted 1 September 2016

\begin{abstract}
Mechanical joint interfaces widely exist in all kinds of components. Their properties are extremely complex as there are a variety of factors affecting contact characteristics such as material, surface roughness, processing method and heat treatment. This study investigated the static mechanical performances of joint interface under cyclic compression loads. A new joint model was established and described as one continuum with three layers of materials, wherein the joint interface was assumed as a layer of material with thickness $h_{\mathrm{c}}$. Then the corresponding theoretical expressions of Total Equivalent Elastic Modulus $\left(E_{t}\right)$ and Interface Equivalent Elastic Modulus $\left(E_{i}\right)$ were proposed. A series of cyclic compression experiments were conducted. And the effects of loading stress, number of cycles and surface roughness on $E_{t}$ and $E_{i}$ was discussed respectively. Results indicated that $E_{t}$ increases first and then levels off with the stress increasing. $E_{t}$ approaches a steady state with the increasing number of cycles. And the modulus decreases with the increase of surface roughness of the joint interface. In addition, $E_{i}$ is a function of $E_{t}$ and the thickness of joint interface $h_{\mathrm{c}}$. The cumulative plastic strain occurs with a repeated loading on the joint interface.
\end{abstract}

Key words: Joint interface / total equivalent elastic modulus / interface equivalent elastic modulus / surface roughness

\section{Introduction}

A machine tool is often made up of many parts. Joint interfaces are produced from the connection of a series of components. Joint interfaces bring about $60 \%-80 \%$ of the total stiffness, $85 \%-90 \%$ of the total static deformation, and $90 \%$ of the total damping in a whole machine tool $[1,2]$. Therefore joint interfaces have great effects on static and dynamical characteristics of machine tools.

As for researches on the contact model of joint interface, Greenwood and Williamson [3] adopted Gaussian distribution to simulate the asperity distribution on contact surface and proposed the classic GW contact model based on Hertz contact theory. Their model laid a milestone for theories concerning rough surface contact [4] and remains widely accepted today. Afterwards some extended models such as WA model [5] and OA model [6] were also proposed. Based on fractal geometry, Majumdar and Bhushan [7] introduced geometrical thoughts of mathematical analysis into the contact theory and proposed the surface fractal model. This model

${ }^{a}$ Corresponding author: yjzeng@bjut.edu.cn is scale-dependent and ignores interactions of asperities. Some researcher such as Zhao [8] studied the normal contact stiffness between two cylinders' joint interfaces using the fractal model. The ultrasonic wave response from a contact interface was modeled first by Kim [9] to investigate the elastoplastic nature of asperity. Following this method, Pecorari [10] used the ultrasonic device to measure the static and dynamic interfacial stiffness. To investigate the deformation model of asperities, Zavarise [11] reviewed and compared elastic [3], plastic [12] and elastoplastic [13] models and demonstrated the importance of plasticity index. As the separation process of compacted interfaces is similar to the fracture process of structure or material, many researchers utilize cohesive zone models (CZMs) to investigate joint interfaces. The common CZMs are polynomial model [14], exponential model [15], trapezoid model [16] and bilinear model [17]. Based on a CZM, Sui [18] took the joint interface as a three-layer structure, in which the upper and lower layers are machine material while the middle is a layer of cohesion. Recently, some new ideas for joint surfaces occured. Nassar [19,20] proposed an improved stiffness model for bolted joints 


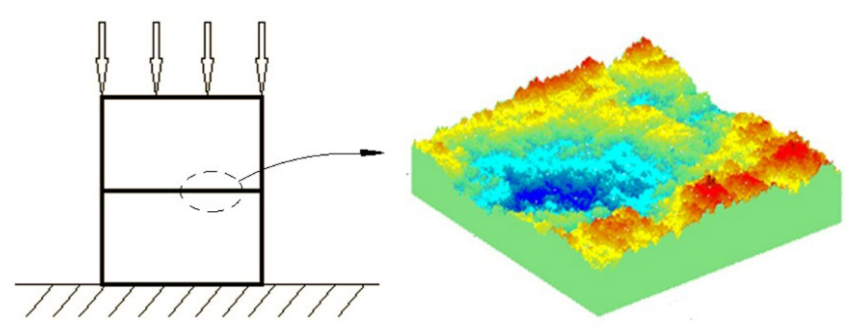

Fig. 1. Joint interface.

and later he investigated the nonlinear deformation behavior of clamed bolted joints under a separating service load. Li [21] presented a new method of dealing with the contact stiffness at interface. His mathematical model included rough surfaces, and was divided into smooth sections with the same length and interface section without thickness. Tian [22] put forward a new dynamic modeling method for fixed joint interfaces based on the hypothesis of virtual material. Kudish [23] obtained an exact solution of the problem for the problem of singly connected contact regions, expressed in the form of a series of Chebyshev polynomials. Hayashi [24] performed a contact analysis on anisotropic materials with the surface stress and surface elasticity taken into consideration.

On the micro-level, the joint interface is constituted by two rough surfaces on which there are quantities of randomly distributed asperities [4]. The properties of joint interface are extremely complex because there are many factors affecting the contact characteristics,such as material, surface roughness, processing method and heat treatment. Therefore, the mechanical properties of joint interfaces are not fully understood.

This paper investigates the mechanical performances joint interface which with different surface roughness through cyclic compression experiments. It establishes a new joint interface model where the joint is described as one continuum with three layer of materials. The corresponding equivalent elastic modulus $E_{t}$ and $E i$ are put forward and their influence factors such as the surface roughness, cycle times and stress are explored separately. Finally, the contact mechanism of joint interface is discussed.

\section{Experiment and results}

The study object of this paper is a flat joint interface (Fig. 1). Both the top and bottom surfaces of specimens are planar. The upper surface is subjected to a uniform pressure and the bottom surface is clamped in testing machine. Mechanical behavior of the couple of the joint interfaces under axial force was investigated.

Table 1 and Figure 2 show test samples with various values of surface roughness and dimensions. The samples are divided into five groups including one group of continuum samples (Group A) and four groups of joint interface samples (Group B-E), their diameters are $10 \mathrm{~mm}$. For Group B-E, the surface roughness is Ra3.2, Ra1.6,
Table 1. Sample groups.

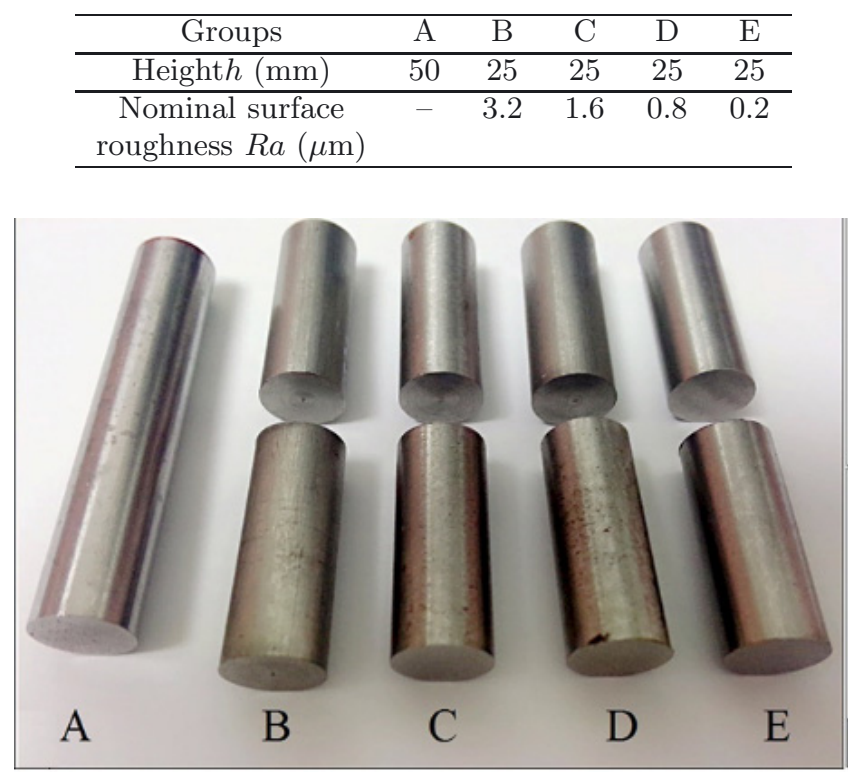

Fig. 2. Dimensions of specimens.

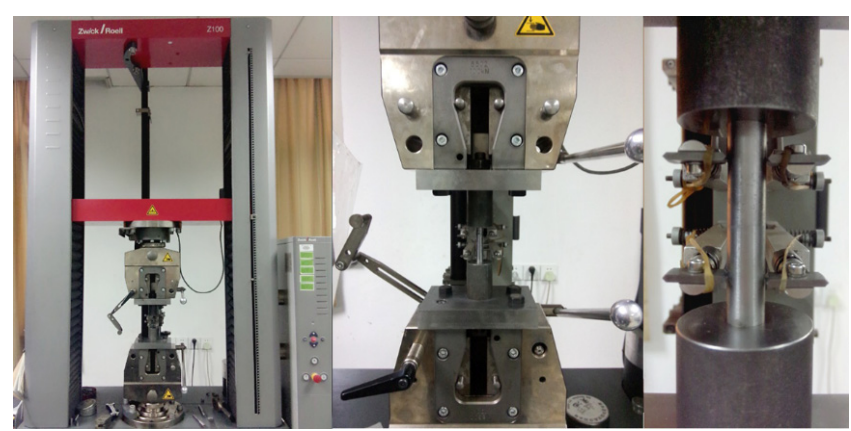

Fig. 3. Zwick/Roell testing machine.

$R a 0.8$ and $R a 0.2$, respectively, and each group contains three pairs of joint interface samples. The samples are made of 45 steel and their yield strength is $355 \mathrm{MPa}$.

A compression testing machine (Zwick100) was used to apply the cyclic axial compression force on samples (Fig. 3). A pair of high-precision extensometers with the gauge length of $30 \mathrm{~mm}$ was used to measure the strain, and the precisions of the extensometers reached the first class. The cyclic force is $12 \mathrm{kN}$, which equals to $75 \%$ of the specimen's proof load so that all the components are just in the range of elastic deformation. Besides, the number of cyclic loading $n=50$ and the loading rate $v=20 \mathrm{~mm} \cdot \mathrm{min}^{-1}$. A pair of samples were stacked up and placed axially between the upper and lower pressure heads. Thus two surfaces contact to form a joint, with the contact interface in the middle of two extensometers.

Experiment results are shown in Figures 4 and 5. Figure 4 shows that stress and strain $(\sigma \sim \varepsilon)$ have a linear relationship in the Group A, and the linear elastic modulus is $210 \pm 0.02 \mathrm{GPa}(n=3)$. Figure 5 shows cyclic $\sigma \sim \varepsilon$ curves of Group B-E with the number of cycles $n=50$, each group contains three pairs of samples. 


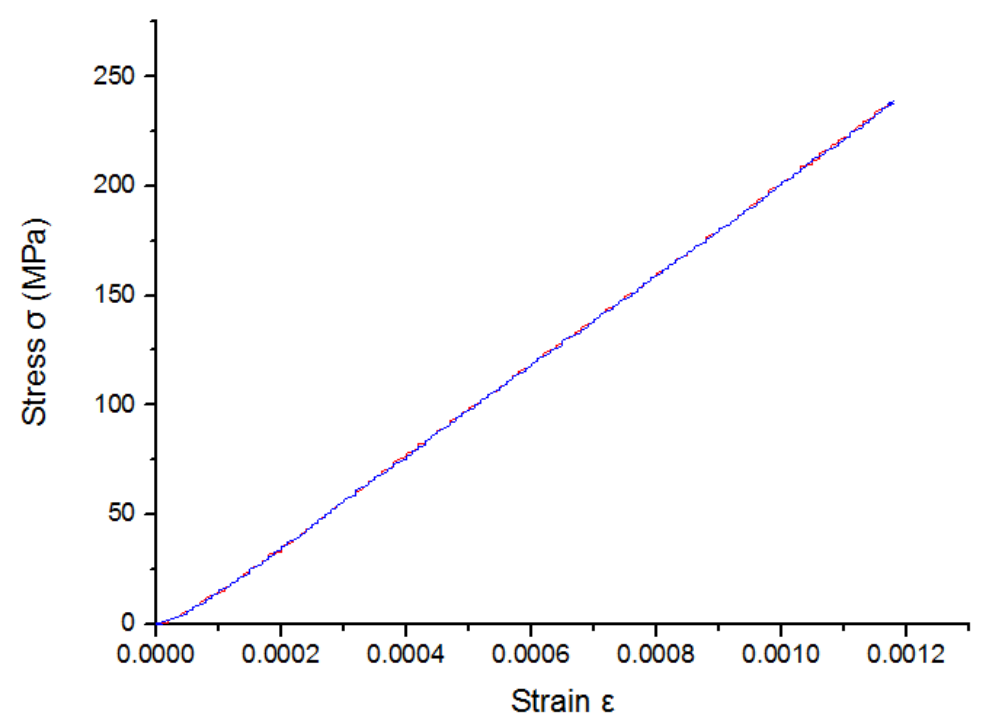

Fig. 4. Compression tests of the Group A.
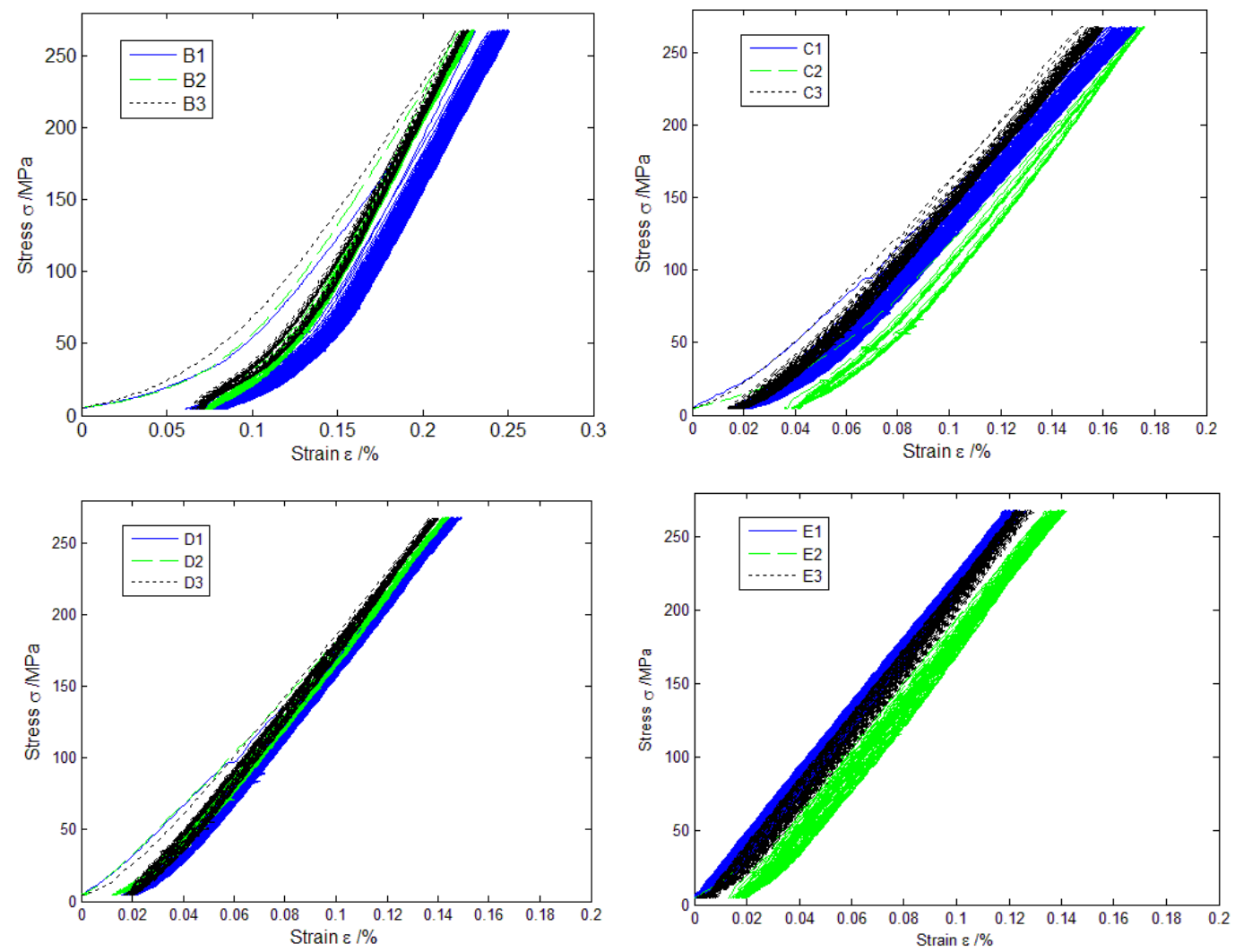

Fig. 5. Compression tests of interface samples.

For each group in Figure 5, the data acquisition system processes stress and strain signals to form three groups of $\sigma \sim \varepsilon$ data, and transmits them to the PC. Through mean processing based on Matlab, the average $\sigma \sim \varepsilon$ curves of Group B-E can be calculated (Fig. 6). This mean method can eliminate the errors of the testing machine and sampes, and include all information of original. The $\sigma \sim \varepsilon$ curves in Figure 6 show nonlinear characteristics. Furthermore, the more nonlinearity increases with the increases of roughness, and the curves develop towards direction of growing strain with the increase of cycle number. The cyclic compression results indicate that the $\sigma \sim \varepsilon$ curves feature nonlinear elasticity. And the main cause of this nonlinearity is the deformation of concave and convex on contact surfaces during the loading process. 


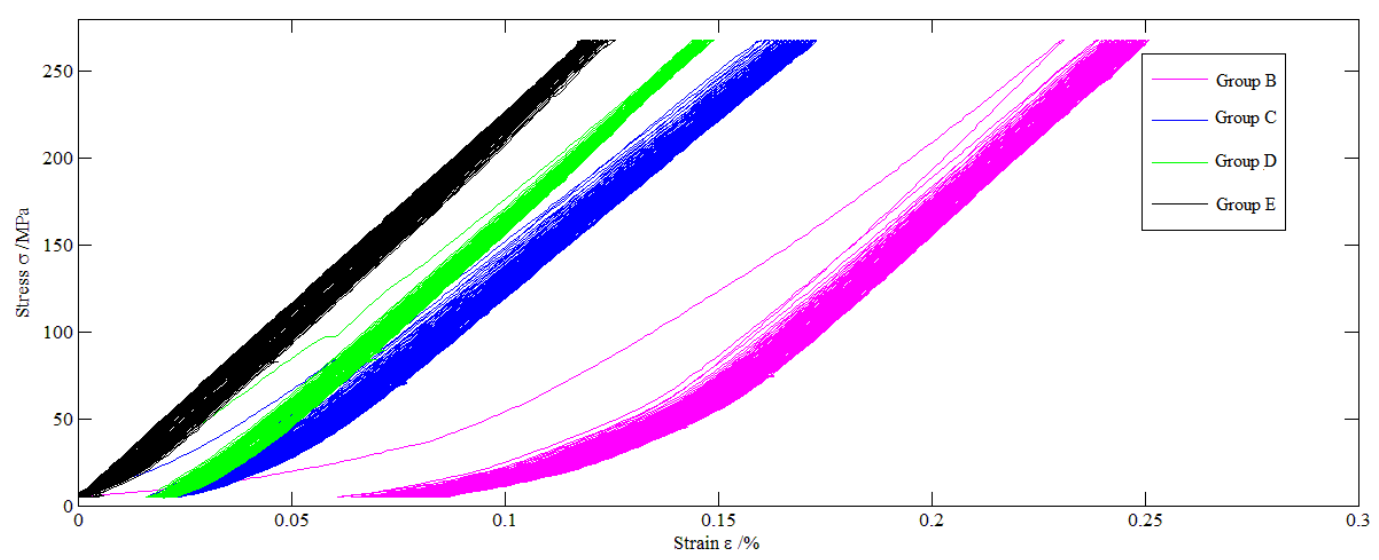

Fig. 6. Compression tests of interface samples.

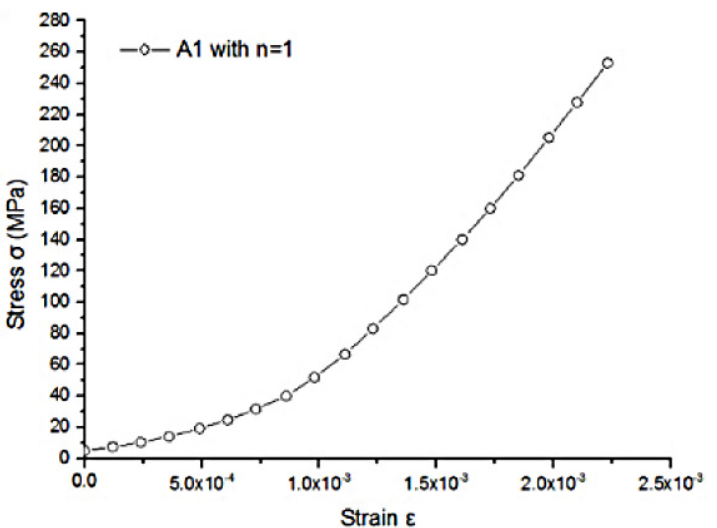

(a)

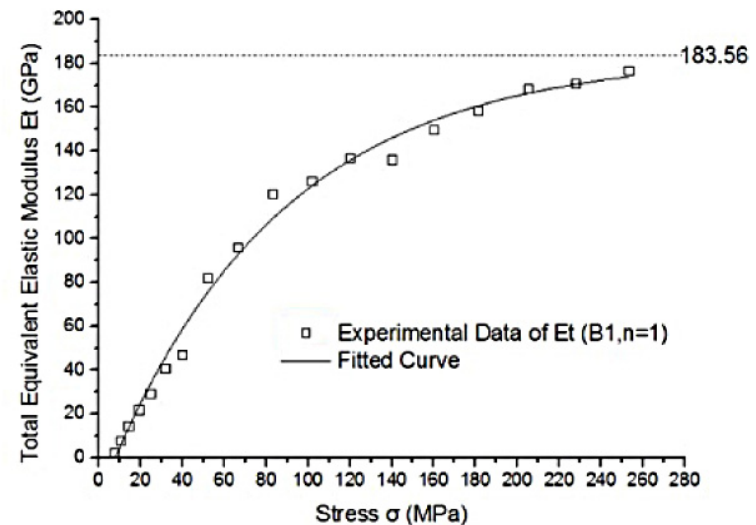

(b)

Fig. 7. Total equivalent elastic modulus of Group B $(n=1)$. (a) $\sigma \sim \varepsilon$ urve, (b) $E_{t} \sim \varepsilon$ curve.

\section{Discussions}

\subsection{Total equivalent elastic modulus}

The slope of $\sigma \sim \varepsilon$ curve in elastic stage represents elastic modulus. For the continuum model, its elastic modulus is a constant, but for the joint surface model, the nonlinear characteristic of $\sigma \sim \varepsilon$ curve represent that its elastic modulus is a variable. In the first loading process, the $\sigma \sim \varepsilon$ curve behaves nonlinear which means the total equivalent elastic modulus is not a constant. $E_{t}$ can be calculated according to the following equation:

$$
\mathrm{d} E_{t}=\mathrm{d} \sigma / \mathrm{d} \varepsilon_{l}
$$

The data indicate that there is an exponential relationship between $E_{t}$ and the stress. Then the following exponential function is adopted to perform a fitting on the experimental data:

$$
E_{t}=y_{0}+A_{1} e^{-\sigma / t}
$$

The fitting function obtained is as follows:

$$
E_{t}=183.56-201.82 e^{-\sigma / 83.34}
$$

where $y_{0}=183.56, A_{1}=-201.82, t=83.34$, and correlation coefficient $R=0.999116$. The asymptotic line $y=y_{0}$ means that $E_{t}$ tends to terminate with the increases of load. According to Equation (2), fifty modulus curves and the corresponding fitting functions are gained (Fig. 8).

Figure 8 shows that $E_{t}$ is no longer a constant, but has a nonlinear relationship with stress for the joint interface model with certain $R a$. i.e. $E_{t}=f(\sigma)$. The development process of $E_{t}$ is divided into three stages, the rapidly increasing stage, slowly increasing stage and the stable stage. At the initial stage, The machined joint interface will be subject to plastic deformation [4], the surface asperities deform largely because their contact areas are small and the contact pressure are relative great. As the stress increasing, the deformations of asperities decrease and their contact areas expand. which is a reason why the total equivalent elastic modulus is a nonlinear function of stress, rather than a constant.

Besides, $E_{t}$ curves develop to intense from sparse. As the number of cycles $n$ changes, the function curve of total equivalent elastic modulus gradually rises and enters into a stable state. This development reflects the process of plastic deformation of the joint interface. Under the action of repeated loads, asperities on the contact surface interact with each other. After multiple loading 

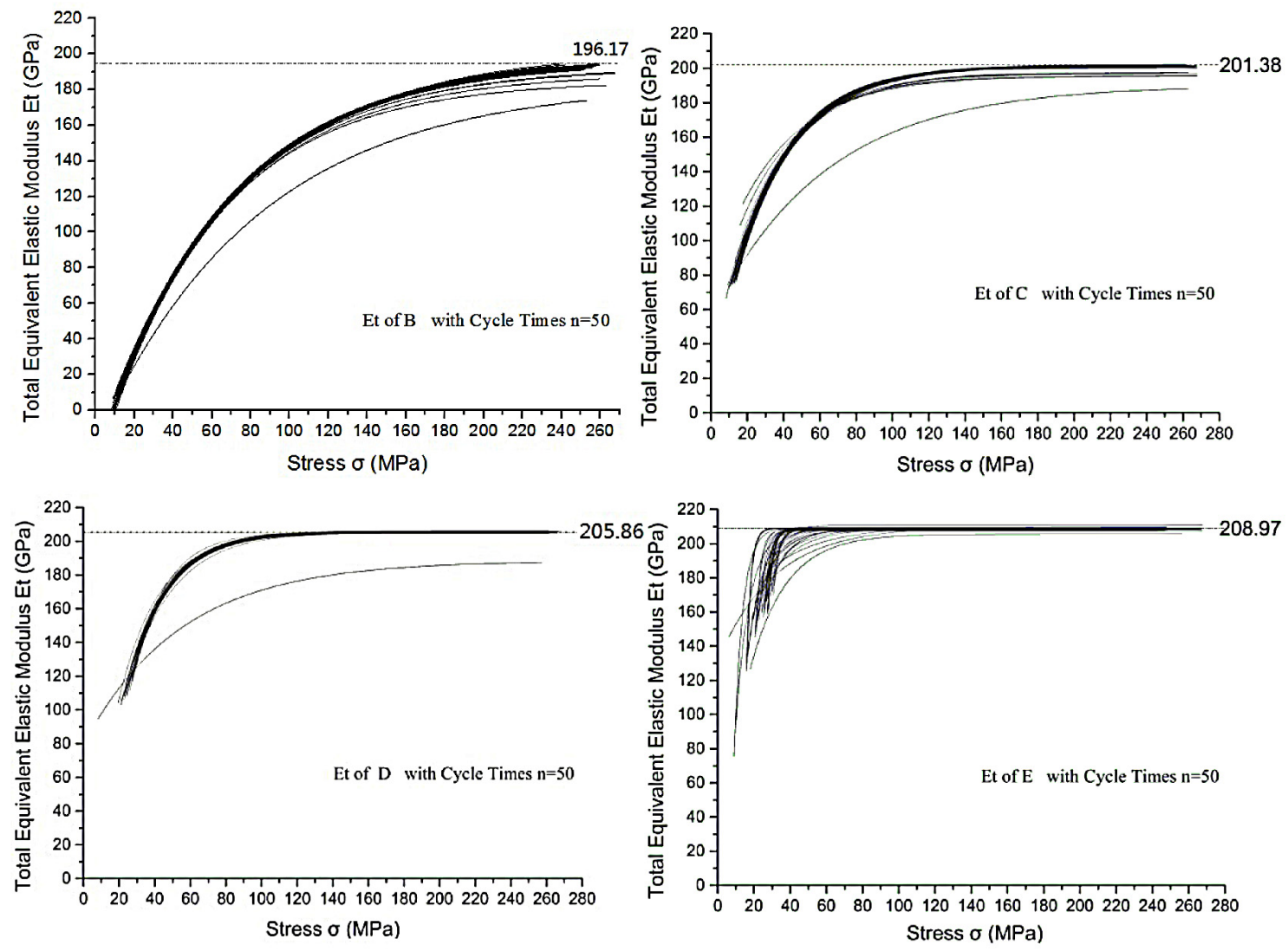

Fig. 8. Total equivalent elastic modulus of Group B-E $(n=50)$.

time after time, the plastic deformations of the joint interface were basically completed and faded away. Then the total equivalent elastic modulus went to the stable state. The corresponding stresses for $E_{t}$ in stable phase $\sigma_{R a 3.2}>\sigma_{R a 1.6>}>\sigma_{R a 0.8}>\sigma_{R a 0.2}$, and the cycle times $n_{R a 3.2>} n_{R a 1.6>} n_{R a 0.8}>n_{R a 0.2}$. This phenomenon dues to the amplitudes of asperities and their plastic deformation.

We define $E_{t}^{s}$ as an eigenvalue to describe the total equivalent elastic modulus in stable state:

$$
E_{t}^{s}=\sum_{i=21}^{50} y_{0-i} / 30
$$

where $y_{0-i}$ represents the value of $y_{0}$ in fitting function Equation (2) when the number of cycles $n=$ $21,22, \ldots, 50$, thus $E_{t-B}^{s}=196.17 \pm 2.34 \mathrm{GPa}$. The stable values of total equivalent elastic modulus $E_{\mathrm{t}}^{s}(\sigma$ is large enough, $n>20$ ) were obtained for each group according to the above analyses, as shown in Table 2 .

It can be observed that the stable extremum of the total equivalent elastic modulus declines with the increasing roughness, i.e. $E_{t-B}^{s}<E_{t-C}^{s}<E_{t-D}^{s}<E_{t-E}^{s}<E_{0}$. It is because those higher asperities on the joint interface cause a larger plastic deformation. In contrast, the plastic deformation of lower asperities on the joint interface is smaller, and the total equivalent elastic modulus increases correspondingly. In general, many factors con-
Table 2. Total equivalent elastic modulus with various $R a$.

\begin{tabular}{ccc}
\hline Groups & Surface roughness on interface & $E_{t}^{s} / \mathrm{GPa}$ \\
\hline B & Ra3.2 & $196.17 \pm 2.34$ \\
C & Ra1.6 & $201.38 \pm 1.01$ \\
D & Ra0.8 & $205.86 \pm 1.04$ \\
E & Ra0.2 & $208.97 \pm 0.51$ \\
\hline
\end{tabular}

tribute to $E_{t}$ such as loading stress, cycle number and surface roughness of joint interface.

\subsection{Interface equivalent elastic modulus theory}

Two rough surfaces contact to form a joint interface (Fig. 9), it is called as a segment model. If the joint interface is treated as one layer of continuous material with the thickness of $h_{\mathrm{c}}$, the segment model turns into a continuum model with three layers of materials, then this model is treated as a joint interface model. The asperities on joint interfaces are supposed to be continuous, homogeneous, and isotropic. The elastic modulus of interface material is called interface equivalent elastic modulus $E_{i}$. The research scope $l$ in Figure $9 \mathrm{~b}$ is the extensometer's measuring length since the joint interface's strain would be contained in an area of the strain measured by the extensometer. We define the elastic modulus of the test section's material which contains joint interfaces as total equivalent elastic modulus $E_{t}$. 


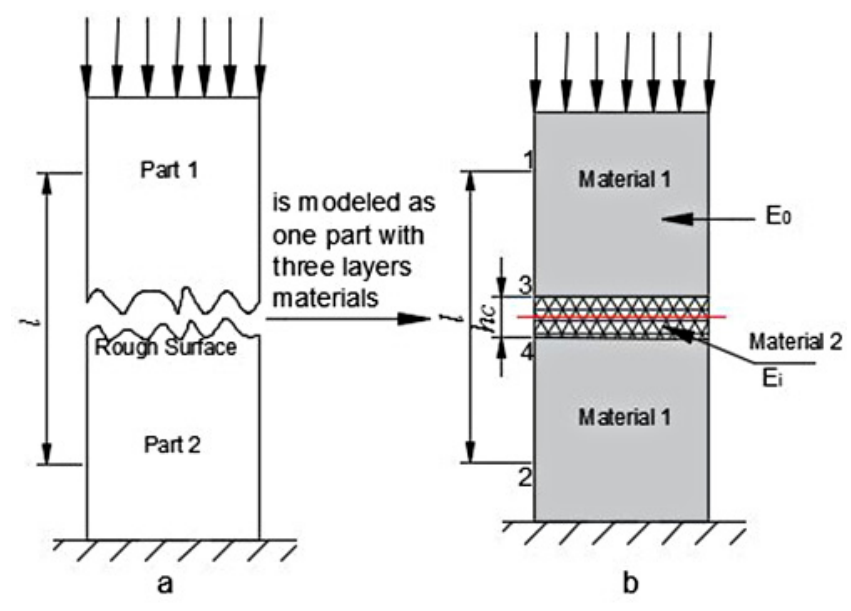

Fig. 9. Theoretical model of joint interface.

In cyclic compression experiments, the total equivalent strain is $\varepsilon_{l}$. The parameter of surface roughness $R a$ is defined as arithmetical mean deviation of the profile, so the roughness amplitude is $2 R a$. Thus a thickness of joint interface can be defined as $h_{\mathrm{c}}=4 R a$.

According to the joint interface model, $E_{t}, E_{i}$ and $E_{0}$ are calculated by:

$$
E_{t}=\sigma / \varepsilon_{l} ; \quad E_{i}=\sigma / \varepsilon_{h_{c}} ; \quad E_{0}=\sigma / \varepsilon_{0}
$$

The overall deformation relationship within the measuring range of extensometer satisfies the following equation:

$$
\delta_{l}=\delta_{h_{c}}+\delta_{0}
$$

where

$$
\begin{array}{r}
\delta_{l}=\varepsilon_{l} \cdot l=\sigma \cdot l / E_{t}, \quad \delta_{h_{c}}=\varepsilon_{h_{c}} \cdot h_{c}=\sigma \cdot h_{c} / E_{i}, \\
\delta_{0}=\varepsilon_{0} \cdot\left(l-h_{c}\right)=\sigma \cdot\left(l-h_{c}\right) / E_{0}
\end{array}
$$

Equation (7) is established based on the principle of deflections consistency:

$$
l / E_{t}=h_{c} / E_{i}+l-h_{c} / E_{0}
$$

Therefore the expression of $E_{i}$ is

$$
E_{i}=\frac{E_{t} \cdot h_{c} \cdot E_{0}}{E_{0} \cdot l-E_{t}\left(l-h_{c}\right)}
$$

where $E_{t}$ and $h_{\mathrm{c}}$ are variables related to the joint surface, and $E_{0}$ is the content modulus related to the bulk of the sample body. Thus $E_{i}$ is a function of $E_{t}$ and $h_{\mathrm{c}}$.

After the total equivalent elastic moduluss obtained, the interface equivalent elastic modulus can be calculated by Equation (8), where the thickness of joint interface $h_{\mathrm{c}}=4 R a$, the elastic modulus of base material $E_{0}=$ $210 \mathrm{GPa}$, and the measurement range of extensometer $l=30 \mathrm{~mm}$. Results obtained are shown in Table 3 .

In Table 3, there is no remarkable difference between the interface equivalent elastic modulus of each groups by the $t$ test of statistics. The mean interface equivalent
Table 3. Interface equivalent elastic modulus.

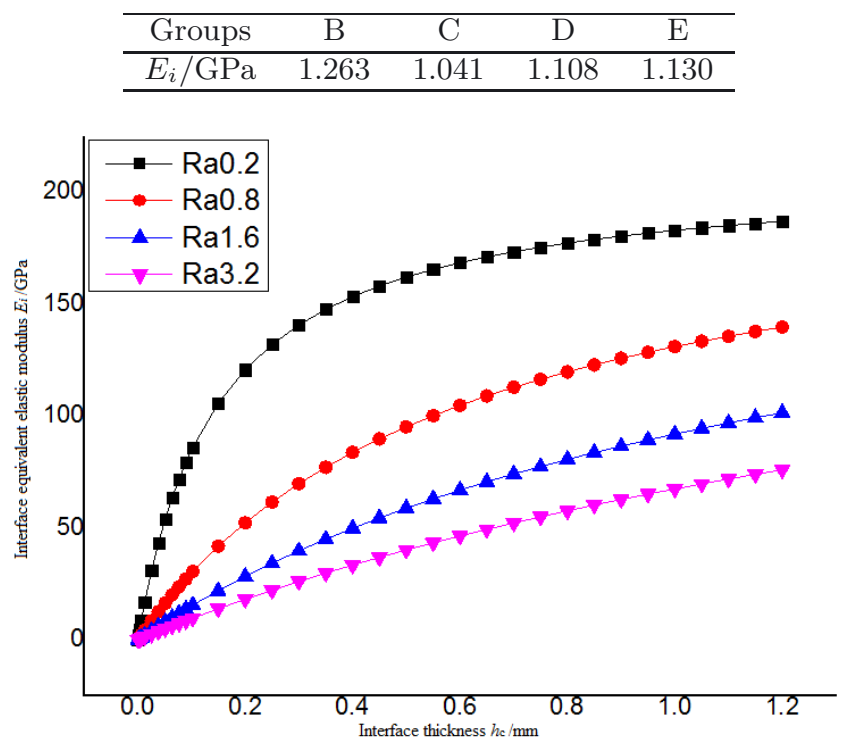

Fig. 10. Interface equivalent elastic modulus.

elastic modulus of four groups is 1.16 Gpa. According to Equation (8), $E_{i}$ is the function of $E_{t}$ and $h_{\mathrm{c}}$, i.e, $E_{i}=f\left(E_{t}, h_{c}\right)$. However the total equivalent elastic modulus varied in the experimental results with the stress changed, and the thickness of joint interface varied with the roughness changed. The reason is that $h_{\mathrm{c}}$ is assumed as $4 R a$, and the machined surface values $R a$ are generally between $0.8 \mu \mathrm{m}$ to $3.2 \mu \mathrm{m}$, so the difference is small. Due to $R a$ can't represent the real surface profile, therefore, to get accurate $h_{\mathrm{c}}$ further studies are needed.

By refering to references, the surface roughness of machine tools' joint is mostly between $0.8-1.6 \mu \mathrm{m}$, and the structure of the metal surface layer is composed of several layers which have different physical and chemical properties and mechanical properties, such as deformation layer, Bielby layer, etc. So $h_{\mathrm{c}}$ should be the thickness of surface to the base material. Figure 10 shows the changes of the $E_{i}$ when $0 \mathrm{~mm}<h_{\mathrm{c}}<1 \mathrm{~mm}$. It indicates that $E_{i}$ decreases with the increases of surface roughness. Equation (8) can be viewed as the experience formula of the mechanical joint design in actual working condition.

\subsection{Contact mechanisms of solid surfaces}

The surface topography was tested through white light interferometer (Fig. 11). It was observed that the surface texture of turning is annular. The real surface roughness before a compression test and after the test is shown in Table 4.

It can be seen from Table 4 that the surface roughness declines obviously after compression. This result suggests the microscopic plastic deformation of the joint interface. This deformation affects the mechanical property of the total equivalent elastic modulus, makes it a nonlinear function of stress, and influences the process of cyclic 

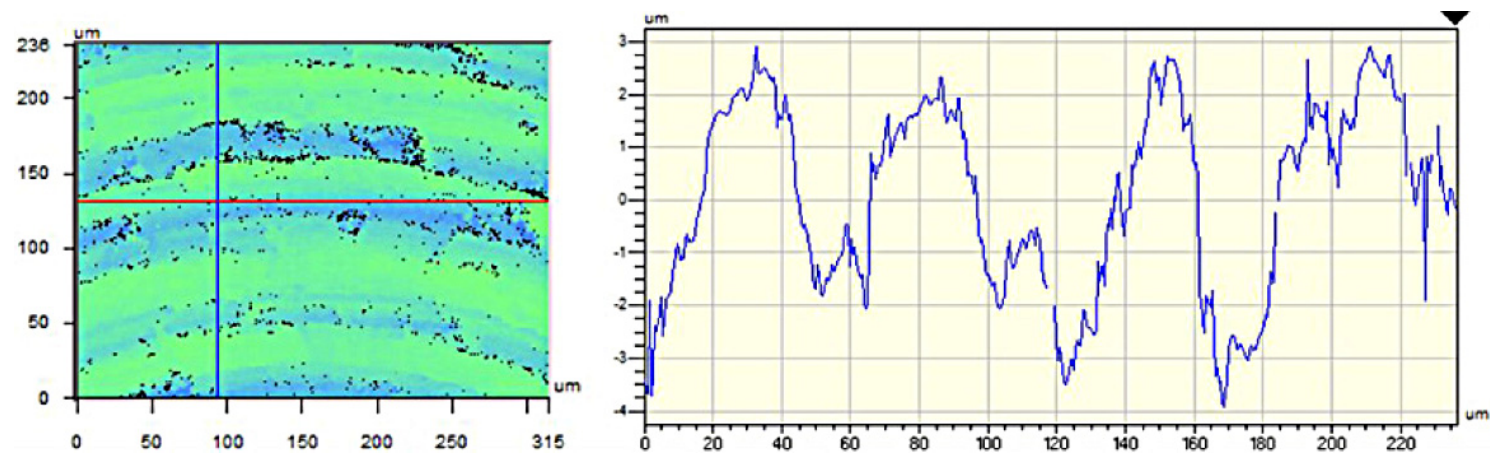

Fig. 11. Surface profile and roughness.

Table 4. Real surface roughness $(\mu \mathrm{m})$.

\begin{tabular}{cccccc}
\hline Group & B & C & D & E \\
\hline \multicolumn{2}{c}{ Nominal roughness } & Ra3.2 & Ra1.6 & Ra0.8 & Ra0.2 \\
\hline \multirow{2}{*}{ Real roughness } & Before Exp. & $3.50 \pm 0.21$ & $1.93 \pm 0.28$ & $0.87 \pm 0.06$ & $0.31 \pm 0.02$ \\
& After Exp. & $3.05 \pm 0.29$ & $1.71 \pm 0.29$ & $0.75 \pm 0.06$ & $0.27 \pm 0.01$ \\
\hline
\end{tabular}

loading. However, the experimental result of the total equivalent elastic modulus indicates that the ratchet phenomenon of the plastic deformation only works for several repeated loads in the beginning, and afterwards the plastic deformation diminishes and the total equivalent elastic modulus stabilizes gradually.

The actual rough surface peak height is generally random distribution, thus the peak point of contact should be based on probability calculation [4], the actual contact area is:

$$
A=m \pi R(z-h)=n \pi R \int_{h}^{\infty}(z-h) \psi(z) d z
$$

Where $n$ is the number of peaks on rough surfaces, $m$ is the number of peaks participating in the contact, $\psi(z)$ is the Gaussian distribution function.

The total bearing load $W$ of all contact peak points is:

$$
\begin{aligned}
W=\frac{4}{3} m E^{*} R^{1 / 2}(z-h)^{3 / 2} & \\
= & \frac{4}{3} n E^{*} R^{1 / 2} \int_{h}^{\infty}(z-h)^{3 / 2} \psi(z) \mathrm{d} z
\end{aligned}
$$

The actual contact area and the bearing load depends on the surface profile curves and the contact state. The average pressure in contact area is:

$$
p_{c}=\frac{W}{A}=\frac{4}{3} \frac{E^{*}}{\pi} \sqrt{\frac{\delta}{R}}
$$

Yielding is determined by the von Mises criterion, expressed as

$$
\sigma_{e q}=\sqrt{\frac{3}{2} S_{i j} S_{i j}}=Y
$$

where $\sigma_{\text {eq }}$ is the von Mises equivalent stress and $S_{\mathrm{ij}}$ represents components of the deviatoric stress tensor.
The contact of rough surfaces is a kind of mixed elastic-plastic system, namely the high peaks produce plastic deformation and the low peaks are in elastic deformation. With load increasing, the normal deformation increases and the peak points with plastic deformation increase either. The surface profile changes under cyclic loading, more asperities occur plastic deformation and the equivalent plastic strain increase, then plastic accumulation happens. Therefore, in actual working condition, for mechanical joints in long-term work stage, the asperities' plastic accumulation behavior on joint interface should be considered, so as to avoid structural relaxation and precision loss.

Discussions in Sections 3.2 and 3.3 show that the metal surface profile has obvious heterogeneity and the stress distribution is complex. Therefore, the interface's thickness $h_{\mathrm{c}}$ and its dynamic model will be the subsequent research topic.

\section{Conclusion}

This study investigated experimentally and theoretically the mechanical joint interface under cyclic compression loads, and established a new joint interface model. Conclusions are as follows:

1. For joint interface model, the total equivalent modulus $E_{t}$ is nonlinear, and its nonlinear degree increases with the increases of surface roughness. With stress and cycle times increasing, $E_{t}$ increases gradually and reaches steady state. Besides, $E_{t}$ decreases with the increase of surface roughness.

2. The joint contact interface is described as an interlayer with thickness of $h_{\mathrm{c}}$, and the theoretical expression of interface equivalent elastic modulus is presented. $E_{i}$ is a function of $E_{t}$ and $h_{\mathrm{c}}$.

3. For the asperities on the contact surfaces, cumulative plastic strain will develop on the contact surfaces under cyclic loading. 
Acknowledgements. This research was financially supported by the National Science and Technology Major Project (2012ZX04010-011).

\section{References}

[1] Y. Ren, C. F. Beards, Identification of 'effective' linear joints using coupling and joint identification techniques, J. Vib. Acoustics-transactions ASME 120 (1998) 331-338

[2] R.A. Ibrahim, C.L. Pettit, Uncertainties and dynamic problems of bolted joints and other fasteners, J. Sound Vib. 279 (2005) 857-936

[3] J.A. Greenwood, J.B.P. Williamson, Contact of nominally flat surfaces, Proceedings of the Royal Society AMathematical Phys. Eng. Sci. 295 (1966) 300-319

[4] K.L. Johnson, Contact mechanics, Cambridge university press, Cambridge, 1985

[5] D.J. Whitehouse, J.F. Archard, The properties of random surfaces of significance in their contact, Proceedings of the Royal Society A-Mathematical Phys. Eng. Sci. 316 (1970) 97-121

[6] R.A. Onions, J.F. Archard, R.A. Onions, et al. The contact of surfaces having a random structure, J. Phys. D 6 (2002) 289-304

[7] A. Majumdar, B. Bhushan, Fractal model of elasticplastic contact between rough surfaces, J. Tribol. 113 (1991) 1-11

[8] H. Zhao, Q. Chen, K. Huang, Fractal model of normal contact stiffness between two cylinders' joint interfaces, Chinese J. Mech. Eng. 47 (2011) 53-5

[9] J.Y. Kim, A. Baltazar, S.I. Rokhlin, Ultrasonic assessment of rough surface contact between solids from elasto plastic loading-unloading hysteresis cycle, J. Mech. Phys. Solids 52 (2004) 1911-1934

[10] C. Pecorari, S.I. Rokhlin, Elasto-plastic micromechanical model for determination of dynamic stiffness and real contact area from ultrasonic measurements, Wear 262 (2007) 905-913

[11] G. Zavarise, M. Borri-Brunetto, Paggi M. On the reliability of microscopical contact models, Wear 257 (2004) 229-245
[12] M.G. Cooper, B.B. Mikic, M.M. Yovanovich, Thermal contact conductance, Int. J. Heat Mass Transfer 12 (1969) 279-300

[13] M.R. Sridhar, M.M. Yovanovich, Review of elastic and plastic contact conductance models-comparison with experiment, J. Thermophys. Heat Transfer 8 (1994) 633640

[14] A. Needleman, A Continuum model for void nucleation by Inclusion debonding, J. Appl. Mech. Transa. ASME 54 (1987) 525-531

[15] A. Needleman, An analysis of tensile decohesion along an interface, J. Mech. Phys. Solids 38 (1990) 289-324

[16] V. Tvergaard, J.W. Hutchinson, The relation between crack-growth resistance fracture process parameters in elastic plastic solids, J. Mech. Phys. Solids 40 (1992) 1377-1397

[17] P.H. Geubelle, J.S. Baylor, Impact-induced delamination of composites: a 2D simulation, Compos. Part-Eng. 29 (1998) 589-602

[18] L.J. Sui, X.G. Tian, T.J. Lu, Static characteristics and cohesive zone model of joint surfaces in machine tools, Chinese J. Solid Mech. (2012) 48-57

[19] S.A. Nassar, A. Abboud, An Improved Stiffness Model for Bolted Joints, J. Mech. Design 131 (2009) 121001-1121001-11

[20] S.A. Nassar, X. Yang, S.V.T. Gandham, et al., Nonlinear Deformation Behavior of Clamped Bolted Joints Under a Separating Service Load, J. Pressure Vessel Technol. 133 (2011) 140-144

[21] H.G. Li, H. Liu, L. Yu, Contact stiffness of rough mechanical joint surface, J. Xi' An Jiaotong University (2011) 69-74

[22] H. Tian, B. Li, H. Liu, et al., A new method of virtual material hypothesis-based dynamic modeling on fixed joint interface in machine tools, Int. J. Machine Tools Manuf. 51 (2011) 239-249

[23] I.I. Kudish, D.K. Cohen, B. Vyletel, Surface roughness and contact connectivity, Proc. Institution Mech. Eng. Part J 228 (2014) 735-744

[24] T. Hayashi, H. Koguchi, Adhesive contact analysis for anisotropic materials considering surface stress and surface elasticity, Int. J. Solids Struct. 53 (2015) 138-147 